\title{
A long-term photometric study of V 1184 Tauri
}

\author{
E. H. Semkov ${ }^{1}$, M. K. Tsvetkov ${ }^{1}$, A. P. Borisova ${ }^{1}$, K. Y. Stavrev ${ }^{1}$, P. Kroll ${ }^{2}$, T. Berthold ${ }^{2}$, \\ K. Birkle ${ }^{3,4}, \mathrm{H}$. Mandel ${ }^{4}$, H. Mito ${ }^{5}$, and K. Tarusawa ${ }^{5}$ \\ 1 Institute of Astronomy, Bulgarian Academy of Sciences, 72 Tsarigradsko Shose blvd., 1784 Sofia, Bulgaria \\ e-mail: esemkov@astro.bas.bg \\ 2 Sternwarte Sonneberg, Sternwartestr. 32, 96515 Sonneberg, Germany \\ 3 Max-Planck-Institut für Astronomie, Königstuhl 17, 69117 Heidelberg, Germany \\ 4 Zentrum für Astronomie Heidelberg, Landessternwarte, Königstuhl 12, 69117 Heidelberg, Germany \\ 5 Kiso Observatory, Institute of Astronomy, School of Science, the University of Tokyo, Mitake-mura, Kiso-gun, \\ Nagano-ken 397-0101, Japan
}

Received 14 December 2007 / Accepted 21 February 2008

\section{ABSTRACT}

\begin{abstract}
Aims. The main purpose of our investigation is to construct the long-time light curve of the PMS star V 1184 Tau. We consider the study of the photometrical variability of PMS stars as very important for understanding stellar evolution. The unusual photometric variability of V 1184 Tau was reported in previous studies as well, but the nature of the observed deep minima is still under discussion. Methods. We present recent data from CCD photometry and from archival photographic plates. The photometric BVRI data presented in this paper were collected from November 2005 to November 2007. To construct the historical light curve of V 1184 Tau, a search for archived photographic observations in the Wide-Field Plate Database (WFPDB) was made. As a result, 412 photographic plates were found containing the field of V 1184 Tau. A part of the plates were scanned at our request and a magnitude estimation was made of V 1184 Tau.

Results. Our recent photometric data suggest that the star brightness is still near the minimum. Thus the period of strong light variations that started in 2003 continues up to the present, hence more than 4 years. Our data from the archival photographic plates suggest that an unknown minimum of brightness exists in the approximate period 1980-1985. Taking all available photometric and spectroscopic data into account we must reject the hypothesis that V 1184 Tau is an FU Orionis type object. V 1184 Tau is a $\mathrm{G}$ type low-mass star whose spectrum is similar to WTT stars, but its photometric behavior is typical of the UX Orionis variable stars. Assuming the obscuration from orbiting dust clouds as a reason for a deep minimum, we estimate the approximate period of obscurations and the interval between two deep minima. Our calculations give a 25-28 year period between the two minima and approximately 8-10 year duration of the minima.
\end{abstract}

Key words. stars: pre-main sequence - stars: individual: V 1184 Tau

\section{Introduction}

The studies of photometric variability in the pre-main sequence (PMS) stars give us important information for the early stages of stellar evolution. Both classes of PMS stars - the widespread low-mass $\left(M \leq 2 M_{\odot}\right)$ T Tauri Stars (TTSs) and the more massive Herbig Ae/Be Stars (HAEBESs) - show various types of photometric variability. Herbst et al. (1994) defined the three main physical mechanisms causing the brightness variation of PMS stars.

Approximately $25 \%$ of HAEBE stars show strong photometric variability with sudden quasi-Algol drops in brightness and amplitudes up to $3 \mathrm{mag}$ in $V$ band (Natta et al. 1997). During the deep minima of brightness, an increase in polarization and specific color variability are observed. The prototype of this group of PMS objects with intermediate mass named UXORs is UX Ori. The general explanation of its variability is a variable obscuration from orbiting circumstellar clumps of dust or edgeon circumstellar disk (Grinin et al. 1991). An alternative model of UXOR phenomena is based on variable gas accretion from a circumstellar disk (Herbst \& Shevchenko 1999).

The unique PMS object V 1184 Tau discovered by Yun et al. (1997) lies in the field of the Bok globule CB 34. A comparison of CCD frames obtained in 1993 with the Palomar
Observatory plates reveals the increasing brightness of this object of $3.7(R)$. The first assumption of those authors about the nature of V 1184 Tau (CB 34V) is an FU Orionis (FUOR) type of outburst in optical wavelengths. Alves et al. (1997) determined the spectral class of V 1184 Tau as G5 (III-IV), the mass as $2 M_{\odot}$, and the age as $10^{6}$ years. Tackett et al. (2003) discovered a 2.372-day rotation period for V 1184 Tau, suggesting the presence of cool surface spots.

The data presented in this paper are a continuation of our investigation of V 1184 Tau (Semkov 2003, 2004, 2006). Our first photometric and spectroscopic investigation (Semkov 2003) reveals V 1184 Tau as a possible weak-line T Tauri star (WTTS) with an amplitude of $0.6(V)$ and spectral variability. In our second paper (Semkov 2004), the beginning of a new deep minimum of the light curve of V 1184 Tau was reported. In the third paper (Semkov 2006), new data from the optical photometry and spectroscopy of V 1184 Tau in the period of minimum light was presented. Photometric data obtained simultaneously with our investigation were published by Tackett et al. (2003), Barsunova et al. (2006), and Grinin et al. (2008).

The analysis of available photometric data suggests that V 1184 Tau shows two types of variability, produced (1) by rotation of a large cool, spotted surface and (2) by occultation from circumstellar clouds of dust or from features of a circumstellar 
disk. Data from multicolor photometry reveal the variation in color indices with stellar brightness typical of UXORs.

\section{Observations}

The recent photometric $B V R I$ data presented in this paper were collected from November 2005 to November 2007. Our photometric observations were obtained in two observatories with three telescopes: the 2-m Ritchey-Chrétien-Coudé and the 50/70/172 cm Schmidt telescopes of the National Astronomical Observatory Rozhen (Bulgaria) and the 1.3-m Ritchey-Chrétien telescope of the Skinakas Observatory ${ }^{1}$ of the Institute of Astronomy, University of Crete (Greece).

Photometrics CCD cameras with the 2-m RCC and 1.3-m RC telescopes and SBIG ST8 camera with the 50/70 cm Schmidt telescope were used. The technical parameters for the CCD cameras used, observational procedure, and data reduction process are described in Semkov (2003). The mean values of instrumental errors are $0.015(I)$ and $0.019(V)$ for observations made with Photometrics CCD cameras, and $0.022(I)$ and $0.029(V)$ for observations made with the ST-8 CCD camera.

The recent results of our photometric observations of V 1184 Tau are summarized in Table 1. The columns give the date of observation, Julian date, IRVB magnitudes, and telescope used. The I-light curve of V 1184 Tau during the period of our observations (Semkov 2003, 2004, 2006, and the present paper) is plotted in Fig. 1.

\section{Search in the WFPDB}

The construction of the historical light curve of V 1184 Tau could be very important for determining the occultation mechanism. One possibility for correctly determining the period of the deep minima is a search in the photographic plate archives. The recent drop in the light curve of V 1184 Tau was the second discovered minimum after the first in 1951, documented on the plates of the Palomar Observatory Sky Survey.

According to Alves et al. (1997) the magnitude of V 1184 Tau estimated from the red Palomar plate (1951) is $\sim 18^{\mathrm{m}}$. Our $R$ magnitudes obtained in March 2004, August 2005, and August 2007 have similar values, so that the star shows the same brightness as in 1951. The now available regular photometric observations cover only the short period from 2000 to 2007. The BVRI magnitudes of V 1184 Tau in December 1993 and December 1994 estimated by Yun et al. (1997) and in February 1996 estimated by Alves et al. (1997) have values close to our magnitudes in the period of maximum brightness. Therefore, we consider that, for a long period (1993-2003), the star passed through the maximal light. The photometric behavior of the star in the period between the two epochs of minima is a subject of our search in the astronomical plate archives.

The Wide-Field Plate Database (Tsvetkov 2006) contains (1) a catalog of all known archives of wide-field $\left(\geq 1^{\circ}\right)$ plates and (2) a merged catalog of wide-field plates. Our search in the database was limited to clear telescope aperture $\geq 40 \mathrm{~cm}$. The results from our detailed examination of the catalog are summarized in Table 2. We found 412 archival photographic observations in the plate collections of 12 telescopes. In addition, one of us (K. B.) found two plates obtained with the $80 / 120 \mathrm{~cm}$ Schmidt telescope of the Max-Planck-Institut für Astronomy, Heidelberg,

\footnotetext{
${ }^{1}$ Skinakas Observatory is a collaborative project of the University of Crete, the Foundation for Research and Technology - Hellas, and the Max-Planck-Institut für Extraterrestrische Physik.
}

Table 1. IRVB photometric observations of V 1184 Tau.

\begin{tabular}{|c|c|c|c|c|c|c|}
\hline Date & JD & $I$ & $R$ & $V$ & $B$ & Tel \\
\hline 25.11 .2005 & 53700.358 & 16.15 & 17.38 & - & - & Sch \\
\hline 26.11 .2005 & 53701.405 & 16.09 & - & - & - & Sch \\
\hline 21.03.2006 & 53816.324 & 16.77 & 17.99 & 19.53 & - & $2 \mathrm{~m}$ \\
\hline 26.03.2006 & 53816.312 & 16.92 & 18.08 & 19.46 & - & $2 \mathrm{~m}$ \\
\hline 19.04 .2006 & 53845.307 & 16.46 & - & - & - & Sch \\
\hline 21.04 .2006 & 53847.298 & 16.56 & - & - & - & Sch \\
\hline 23.04 .2006 & 53849.260 & 16.51 & 17.70 & - & - & Sch \\
\hline 19.08 .2006 & 53966.611 & 15.13 & 16.85 & 18.40 & - & $1.3 \mathrm{~m}$ \\
\hline 21.08 .2006 & 53968.611 & 15.31 & 17.03 & 18.55 & - & $1.3 \mathrm{~m}$ \\
\hline 22.08 .2006 & 53969.607 & 15.20 & 16.88 & 18.47 & 20.18 & $1.3 \mathrm{~m}$ \\
\hline 23.08 .2006 & 53970.608 & 14.92 & 16.65 & 18.19 & - & 1.3 \\
\hline 25.08 .2006 & 53972.613 & 14.65 & 16.26 & 17.73 & - & 1.3 \\
\hline 26.08 & 53973 & 14.76 & - & 17.81 & - & $1.3 \mathrm{~m}$ \\
\hline 29.0 & $53 c$ & 14.28 & & & - & $1.3 \mathrm{~m}$ \\
\hline 30.08 & 5397 & 14.42 & 15.97 & 17.37 & - & $1.3 \mathrm{~m}$ \\
\hline 31.0 & $5397 \xi$ & 14.38 & 15.92 & 17.32 & 19. & $1.3 \mathrm{~m}$ \\
\hline 03.0 & 53981. & 14.83 & 16.43 & 17.84 & 19. & $1.3 \mathrm{~m}$ \\
\hline 07.09 & 53985.517 & 16.04 & 17.53 & 18.86 & - & $1.3 \mathrm{~m}$ \\
\hline 08.09.2006 & 53986.535 & 16.15 & 17.69 & 19.11 & - & $1.3 \mathrm{~m}$ \\
\hline 09.09 .2006 & 53987.544 & 16.27 & 17.82 & 19.24 & - & $1.3 \mathrm{~m}$ \\
\hline 10.09 .2006 & 53988.543 & 16.32 & 17.76 & 19.15 & - & $1.3 \mathrm{~m}$ \\
\hline 13.09 .2006 & 53991.562 & 16.44 & 17.80 & 19.13 & - & $1.3 \mathrm{~m}$ \\
\hline 14.0 & 5399 & 16.44 & 17.81 & 19.14 & - & $1.3 \mathrm{~m}$ \\
\hline .2006 & 5399 & 15.70 & 17.52 & 19.04 & - & $1.3 \mathrm{~m}$ \\
\hline & 539 & 15.06 & 16.88 & 18. & - & $1.3 \mathrm{~m}$ \\
\hline 2006 & 539 & 14.62 & 16.42 & 99 & - & $1.3 \mathrm{~m}$ \\
\hline & & 14.84 & 16.64 & & - & $1.3 \mathrm{~m}$ \\
\hline & & 14.97 & 16.65 & & - & $1.3 \mathrm{~m}$ \\
\hline 06 & 539 & 15.04 & - & & - & $1.3 \mathrm{~m}$ \\
\hline 22.0 & 540 & 15.07 & - & & - & $1.3 \mathrm{~m}$ \\
\hline 23.0 & 540 & 15.15 & 16.93 & 47 & - & $1.3 \mathrm{~m}$ \\
\hline 25.0 & 540 & 15.29 & 17.08 & 18.60 & - & $1.3 \mathrm{~m}$ \\
\hline 26.0 & 5400 & 15.18 & 17.01 & 18.59 & - & $1.3 \mathrm{~m}$ \\
\hline 27.09 .2006 & 54006.476 & 15.18 & 16.95 & 18.46 & - & $1.3 \mathrm{~m}$ \\
\hline 29.09 .2006 & 54008.483 & 15.13 & 16.92 & 18.47 & - & $1.3 \mathrm{~m}$ \\
\hline 30.09 .2006 & 54009.472 & 15.38 & 17.11 & 18.54 & - & $1.3 \mathrm{~m}$ \\
\hline 05.10 .2006 & 54015.452 & 15.32 & 17.12 & 18.65 & - & $1.3 \mathrm{~m}$ \\
\hline 19.10 .2006 & 54027.509 & 15.91 & 17.27 & - & - & Sch \\
\hline 19.10.2006 & $5402 \varepsilon$ & 16.20 & 17.37 & 19.02 & - & Sch \\
\hline & 540 & 16.31 & 17.75 & - & - & Sch \\
\hline 06 & 54057 & 16.03 & 17.43 & 18.8 & - & Sch \\
\hline & & 16.09 & 15.47 & 18. & - & Sch \\
\hline & & 16.17 & - & - & - & Sch \\
\hline & 540 & 15.94 & 17.27 & & - & $2 \mathrm{~m}$ \\
\hline & 540 & 15.76 & 17.17 & & 20. & $2 \mathrm{~m}$ \\
\hline & 540 & 15.56 & 16.99 & 18. & - & Sch \\
\hline 2007 & 5415 & 16.42 & - & - & - & Sch \\
\hline 25.02.2007 & 54157.353 & 16.47 & - & - & - & Sch \\
\hline 08.04.2007 & 54199.323 & 16.65 & 17.61 & - & - & $2 \mathrm{~m}$ \\
\hline 09.04.2007 & 54200.265 & 16.95 & 17.97 & 19.26 & - & $2 \mathrm{~m}$ \\
\hline 10.04 .2007 & 54201.286 & 16.61 & - & - & - & Sch \\
\hline 02.08 .2007 & 54314.603 & 16.99 & - & 19.77 & - & 1.3 \\
\hline 15.08 .2007 & 54327.579 & 16.96 & - & 19.27 & - & $2 \mathrm{~m}$ \\
\hline 16.08 .2007 & 54328.577 & 17.07 & - & 19.35 & - & $2 \mathrm{~m}$ \\
\hline 17.08 .2007 & 54329.569 & 16.96 & - & 19.24 & - & $2 \mathrm{~m}$ \\
\hline 18.08 .2007 & 54330.565 & 17.15 & 18.17 & 19.32 & - & $2 \mathrm{~m}$ \\
\hline 06.11 .2007 & 54411.390 & 16.17 & 17.28 & 18.66 & - & $2 \mathrm{~m}$ \\
\hline 08.11 .2007 & 54412.545 & 15.78 & - & - & - & $2 \mathrm{~m}$ \\
\hline
\end{tabular}

at Calar Alto Observatory. (The plate catalog of this telescope is still not included in the WFPDB.)

The first results from the plate archives of Kiso, Sonneberg, Heidelberg, Rozhen, and Konkoly observatories are reported in the present paper. The digitized plates from the Palomar Schmidt telescope, available via the website of the Space 


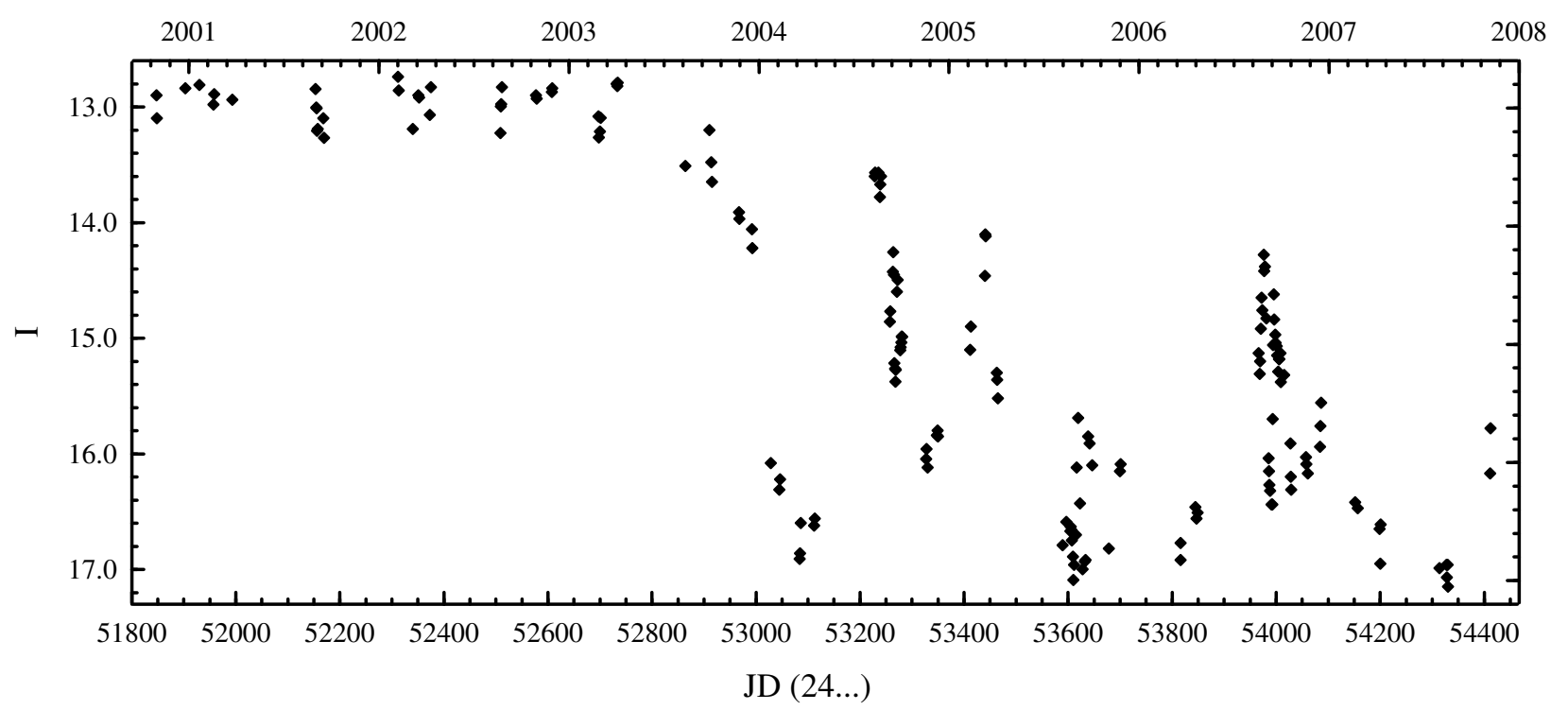

Fig. 1. I light curve of V 1184 Tau.

Table 2. Archival photographic plates of V 1184 Tau found in WFPDB.

\begin{tabular}{lrlrlr}
\hline \hline Observatory & $\begin{array}{r}\text { Telescope } \\
\text { aperture }(\mathrm{cm})\end{array}$ & $\begin{array}{l}\text { Telescope } \\
\text { type }\end{array}$ & $\begin{array}{r}\text { Number } \\
\text { of plates }\end{array}$ & $\begin{array}{l}\text { Band } \\
\text { pass }\end{array}$ & $\begin{array}{r}\text { Range } \\
\text { of years }\end{array}$ \\
\hline Sonneberg & 40 & Ast. & 273 & $\mathrm{pg}$ & $1938-1993$ \\
Sonneberg & 50 & Sch. & 7 & $\mathrm{pg}$ & $1961-1985$ \\
Heidelberg & 40 & Ast. & 50 & $\mathrm{pg}$ & $1900-1980$ \\
Palomar & 122 & Sch. & 25 & $\mathrm{pg} \mathrm{BVRI}$ & $1951-1998$ \\
Harvard & 61 & Rfr. & 19 & $\mathrm{pg}$ & $1894-1938$ \\
Kiso & 105 & Sch. & 11 & $\mathrm{BR}$ & $1976-1989$ \\
Rozhen & 50 & Sch. & 8 & $\mathrm{pg} \mathrm{B}$ & 1985 \\
Konkoly & 60 & Sch. & 7 & $\mathrm{pg} \mathrm{BR}$ & 1985 \\
Turku & 50 & Sch. & 6 & $\mathrm{pg}$ & $1941-1945$ \\
Asiago & 67 & Sch. & 1 & $\mathrm{pg}$ & 1969 \\
Asiago & 40 & Sch. & 2 & $\mathrm{pg} \mathrm{B}$ & 1985 \\
Upsala & 100 & Sch. & 3 & $\mathrm{pg}$ & $1983-1985$ \\
\hline Total & & & 412 & & $1894-1998$ \\
\hline
\end{tabular}

Telescope Science Institute, are used, too. The most important for our investigation are the photographic plates from the Sonneberg and the Heidelberg observatories. The plates from both archives cover a wide time interval with regular observations. Unfortunately, most of the plates were obtained with the $40 \mathrm{~cm}$ astrographes, and V 1184 Tau is only detectable in the period of maximal light, just above the plate limit. In the period of big light variations, V 1184 Tau was detected on the plates from the big Schmidt telescopes in Palomar, Kiso, and Calar Alto.

The field of Bok globule CB 34 is located at approximately $2^{\circ}$ from the ecliptic, and this region is of interest for the investigators of comets and minor planets. For instance, during the recent return of the comet Halley, it passed at approximately $1^{\circ}$ from the position of V 1184 Tau (October 26, 1985). Some of the photographic observations of the comet made in this period can be very important for brightness estimation of V 1184 Tau. We shall in the near future also try to collect digitized plates of V 1184 Tau from the rest of the archives.

\section{Results and discussion}

Our CCD photometric data (Semkov 2003, 2004, 2006, and the present paper) suggest that, from October 2000 to April 2003, the brightness of V 1184 Tau fluctuated around $I=13^{\mathrm{m}} 0$ with an amplitude of about 0.5 (Fig. 1). Such brightness variations can be seen in the other passbands, too. Since August 2003 a gradual decrease in the star brightness began and the $I$ magnitude of V 1184 Tau decreased with $\sim 4^{\mathrm{m}}$ until March 2004. Periodic low-amplitude variations were observed simultaneously with the deep minima, which is illustrated in Fig. 1.

Another important result from our photometric study is the variation in color indices with stellar brightness. In Fig. 2 we plot the measured color indices $V-I$ and $V-R$ versus stellar magnitude $V$ during the period of our observations. A clear dependence can be seen in the period of maximum light: the star becomes redder as it fades. In our first paper (Semkov 2003), we drew attention to such color variations being typical of stars with large cool spots, whose variability is produced by rotation of the spotted surface (WTTS).

The tendency towards reddening becomes considerable from August 2003. While in the period of maximum brightness the color index $V-I$ varies between $2 \mathrm{~m} 15$ and 2 . 40 , it reaches a value of $3 \mathrm{~m} .22$ in October 2004. From a certain turning point $(V \sim 18.2) \mathrm{V} 1184$ Tau gets bluer, fading further to $V-I=$ 2. 44 in March 2004. Such color variations are also observed for the $V-R$ index (Fig. 2). In this case we suggest that the reddening of the star is produced by the variable extinction from circumstellar environment (type III from the Herbst et al. 1994 

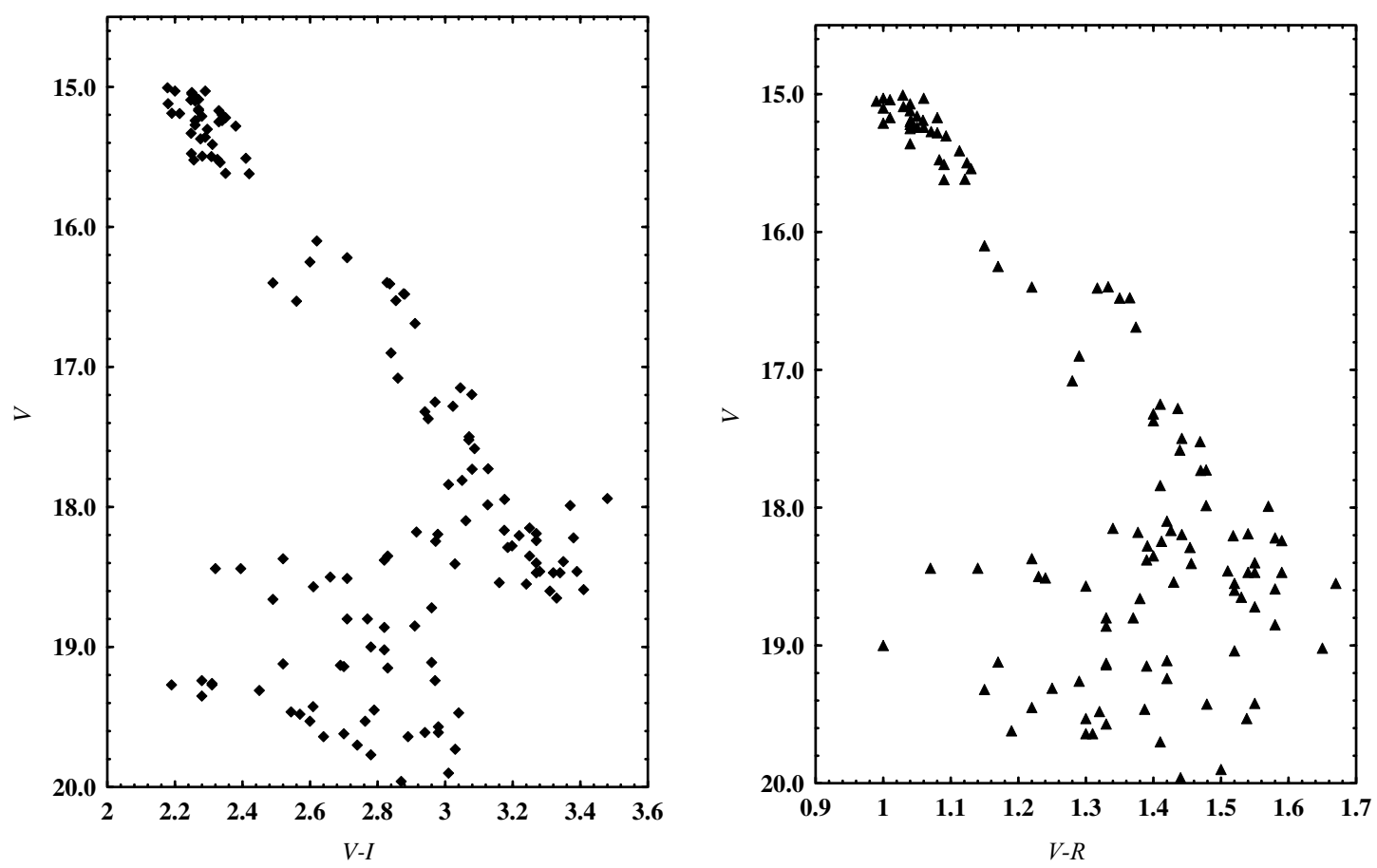

Fig. 2. $V / V-I$ and $V / V-R$ diagrams of $\mathrm{V} 1184$ Tau.

classification). Our photometric observations confirm a "blueing effect" in the minimum light, a typical feature of the PMS stars from UXOR type.

The effect of color reversal (or co-called "blueing") has been described in many studies (Bibo \& The 1990; Grinin et al. 1994; Grady et al. 1995; van den Ancker et al. 1996). According to the model of dust-clump obscuration, the observed color reversal is caused by the scattered light from the small dust grains. Normally the star becomes redder when its light is covered by dust clouds on the line of sight. But when the obscuration rises enough, the part of the scattered light in the total observed light becomes significant and the star color gets bluer. Our photometric data suggest that the $V-I$ index increases in the very deep minimum back to the values $3{ }^{\mathrm{m}} 0-3^{\mathrm{m}} 1$ (Fig. 2). This led us to the assumption of a second turning point at approximately $V \sim 19$. 5, when the star becomes redder again, or the "blueing effect" has finished. The problem for determining this possible second turning point follows from the presence of a short time variability and from the "flare-like" events as observed during the summers of 2005 and 2006 (Fig. 1). To minimize the effect from the different type of variability and to prove our assumption, in the future we will try to collect much more photometric data around the deep minima.

After the search in the WFPDB for plates containing the field of V 1184 Tau, we started a procedure for selecting the plates suitable for photometric measurements. We rejected the plates obtained with a short exposure time, with an inappropriate method of observation (objective prism plates, multiexposure plates, etc.) and with low photometric quality. Some of the plates were rejected because the star was outside of the plate image or lying edge-on. The old plates (from archives of Heidelberg and Sonneberg astrographs) were obtained on less sensitive emulsions, and the plate limit is very low in spite of the long exposure time. Our first selection of plates with good quality for magnitude measurements includes seven plates from the Kiso Schmidt telescope, two plates from the Calar Alto Schmidt telescope, three plates from the Sonneberg Schmidt telescope, three plates from the Bruce astrogrsph in Heidelberg, six plates from the Sonneberg astrogrsph, and nine digitized plates from the archive of the Palomar Schmidt telescope. The plates from the 105/150 Schmidt telescope were digitized with the PDS microdensitometer of the Kiso Observatory. The plates from Heidelberg Observatory were scanned with 2540 dpi resolution, corresponding to a $10 \times 10 \mu \mathrm{m}$ pixel size $\left(0{ }^{\prime} 86 \times 00^{\prime} 86\right.$ for the Schmidt plates and 1'.02 × 1.'02 for the plates from the Bruse astrograph). All Sonneberg plates were scanned with 1200 dpi resolution; plate scales can be found in the "List of Wide-Field Plate Archives" (http: //www.skyarchive.org/ data/alistv25.html). Aperture photometry of the digitized plates used DAOPHOT routines. The UBVRI comparison sequence reported in Semkov (2006) was used as a reference. The results from magnitude estimation of the archival photographic plates are summarized in Table 3.

The plates obtained with the 50/70 cm Schmidt telescope at Rozhen Observatory were inspected visually. Unfortunately, they were made with short exposures (5-6 min) for co-ordinate estimation of Halley Comet, and V 1184 Tau is under the plate limit ( $\sim 16.8 \mathrm{pg})$. The only plate found in the plate collection of Konkoly observatory - No. 2870 (the rest of the plates are not available) - was made with a 5 min exposure time and is not usable for photometry.

As seen from Table 3, most of the photographic plates were obtained in $B / p g$ and $R$ bandpasses. Therefore, we can construct the historical light curves of V 1184 Tau and analyze the photometric behavior of the star only for these two bandpasses (Fig. 3). The first result from our investigation of the archival photographic plates of V 1184 Tau is the presence of an unknown deep minimum in the period 1980-1985. The minimum started between January 1976 and January 1980 and finished between October 1985 and November 1986. All photographic plates obtained in the period January 1980-October 1985 show $B V R$ and $p g$ magnitudes of V 1184 Tau below the minimum values in the period of maximal light. Thus the duration of the discovered minima varies between 6 and 10 years. It is quite 
Table 3. Photometric data from the archival photographic plates of V 1184 Tau.

\begin{tabular}{lllllr}
\hline \hline Observatory & Plate No. & Bandpass & Date & JD (24...) & Magnitude \\
\hline Sonneberg & A 247 & $p g$ & 14.02 .1939 & 29303.33 & $17.0 \pm 0.2$ \\
Palomar & 000416 & $p g$ & 28.11 .1951 & 33958.89 & $20.4 \pm 0.3$ \\
Palomar & 000416 & $R$ & 28.11 .1951 & 33958.90 & $16.8 \pm 0.2$ \\
Palomar & 000427 & $p g$ & 16.12 .1951 & 33978.81 & $20.9 \pm 0.4$ \\
Palomar & 000427 & $R$ & 16.12 .1951 & 33978.82 & $17.8 \pm 0.2$ \\
Heidelberg & B 7411 & $p g$ & 23.01 .1954 & 34766.50 & $>16.7$ \\
Heidelberg & B 9044 & $p g$ & 11.03 .1961 & 37370.50 & $>16.7$ \\
Heidelberg & B 9045 & $p g$ & 11.03 .1961 & 37370.50 & $>16.9$ \\
Sonneberg & A 625 & $p g$ & 18.01 .1963 & 38048.42 & $>17.0$ \\
Sonneberg & A 1589 & $p g$ & 13.11 .1966 & 39443.44 & $16.8 \pm 0.2$ \\
Sonneberg & A 1637 & $p g$ & 14.02 .1967 & 39536.35 & $16.8 \pm 0.3$ \\
Sonneberg & A 2500 & $p g$ & 07.11 .1967 & 39801.59 & $17.0 \pm 0.2$ \\
Sonneberg & A 2504 & $p g$ & 08.11 .1967 & 39802.55 & $16.8 \pm 0.2$ \\
Kiso & 000158 & $R$ & 04.01 .1976 & 42781.94 & $14.4 \pm 0.1$ \\
Kiso & 002569 & $B$ & 18.01 .1980 & 44257.15 & $17.4 \pm 0.2$ \\
Kiso & 002570 & $B$ & 18.01 .1980 & 44257.19 & $17.3 \pm 0.2$ \\
Palomar & 000335 & $V$ & 16.12 .1982 & 45319.83 & $16.0 \pm 0.1$ \\
Palomar & 000370 & $V$ & 07.01 .1983 & 45341.78 & $15.9 \pm 0.2$ \\
Kiso & 004298 & $R$ & 24.01 .1984 & 45724.08 & $16.1 \pm 0.1$ \\
Calar Alto & A 87 & $R$ & 31.07 .1985 & 46278.35 & $15.1 \pm 0.2$ \\
Calar Alto & A 107 & $R$ & 10.10 .1985 & 46348.66 & $16.3 \pm 0.3$ \\
Sonneberg & S 5934 & $p g$ & 21.10 .1985 & 46359.50 & $>17.3$ \\
Sonneberg & S 5945 & $p g$ & 22.10 .1985 & 46360.50 & $>17.6$ \\
Kiso & 004768 & $B$ & 24.10 .1985 & 46363.28 & $19.0 \pm 0.4$ \\
Sonneberg & S 5947 & $p g$ & 25.10 .1985 & 46363.50 & $>17.4$ \\
Palomar & 000932 & $B$ & 05.11 .1986 & 46739.92 & $16.4 \pm 0.2$ \\
Palomar & 000999 & $R$ & 27.12 .1986 & 46791.77 & $14.3 \pm 0.2$ \\
Palomar & 001004 & $I$ & 29.12 .1986 & 46793.75 & $13.3 \pm 0.1$ \\
Kiso & 005322 & $B$ & 22.01 .1987 & 46818.11 & $16.6 \pm 0.2$ \\
\hline & & & & & \\
\hline & & & & & \\
& & & & &
\end{tabular}

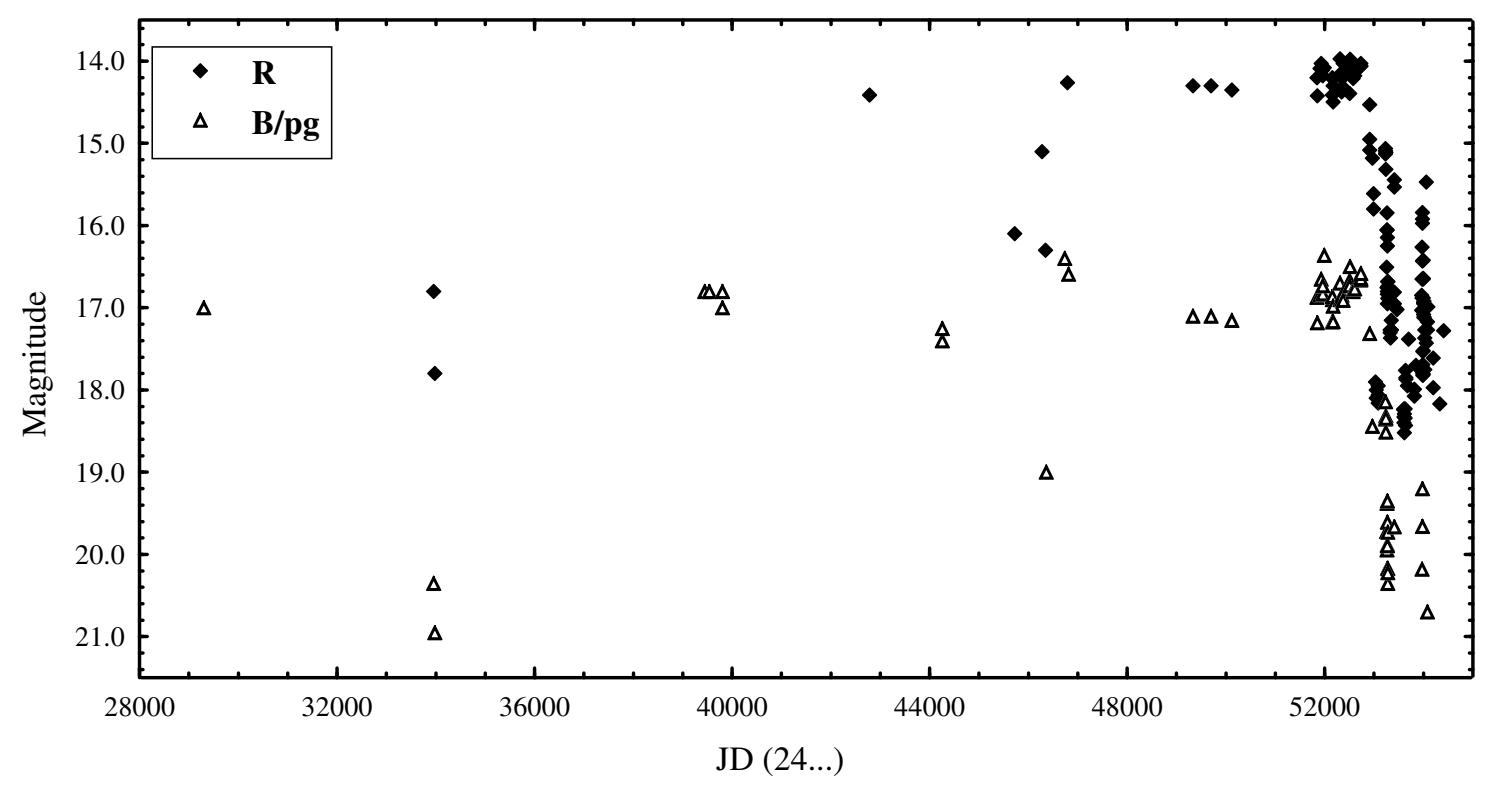

Fig. 3. Historical $R$ and $B / p g$ light curves of V 1184 Tau.

difficult to estimate the duration of the first minimum documented on the plates from the first Palomar Observatory Sky Survey. We only know that in November-December 1951 the star brightness was in a deep minimum. Subsequent observations when available were made in the period 1954-1963 with the $40 \mathrm{~cm}$ astrographs of Heidelberg and Sonneberg observatories. The estimated limit of these observations is 16.7-17.0, and the object was not detected. We know from our CCD observations that, in the maximum light, the $B$ magnitude of the star varies between 16.50 and 17.20; therefore, it is not possible to specify the end of the minimum. The present minimum started in August 2003 and has lasted more than 4 years up to now.

For the 56-year period of observations, three deep minima were observed. Assuming the obscuration from orbiting dust clouds as the reason for a deep minimum, we estimate the approximate period of obscurations and the interval between two 
deep minima. Our calculations give a $25-28$ year period between two minima and approximately an 8-10 year duration of each minimum.

\section{Conclusions}

The unusual photometric behavior of V 1184 Tau has no precise analogies in the PMS stars. At this stage it is most unlikely that V 1184 Tau is a FUOR type variable. No one of the well-studied FUORs shows such a strong brightness decrease or a recurrent outburst in a short time period. Therefore, the variability of V 1184 Tau can be explained as a superposition of two independent phenomena: (1) variability caused by rotation of the spotted surface and (2) occultation from circumstellar clouds of dust or from features of a circumstellar disk. Our multicolor photometric observations suggest that V 1184 Tau can be added to the group of UXOR stars, but no one of the known UXORs shows a short period rotation variability. The typical amplitude for UXOR star is 2-3 mag when the observed amplitude of V 1184 Tau reaches 5 mag in $V$ band. In contrast to the classical UXORs, HAEBE and classical T Tauri stars, the spectrum of V 1184 Tau shows the features typical of WTT stars (Semkov 2003). A possible future study of the rest of the photographic plate archives, along with the next photometric observations, will aim at supplementing of the long-term light curve of V 1184 Tau and determining the duration of the deep minima more precisely. We encourage those observers interested in PMS variables to continue following V 1184 Tau to the end of the present minima. Very important for this study are multicolor photometric observations in the period of minimum light.
Acknowledgements. The authors thank the Director of Skinakas Observatory, Prof. I. Papamastorakis, and Prof. I. Papadakis for the telescope time. The Heidelberg plate-scanning project is financed by the Klaus-Tschira Foundation, Heidelberg under contract No. 00.071.2005. The Digitized Sky Survey was produced at the Space Telescope Science Institute under US Government grant NAG W-2166. The images of these surveys are based on photographic data obtained with the Oschin Schmidt Telescope on Palomar Mountain and the UK Schmidt Telescope. The plates were processed into the present compressed digital form with the permission of these institutions.

\section{References}

Alves, J. F., Hartman, L., Briceno, C., \& Lada, C. J. 1997, AJ, 113, 1395 van den Ancker, M. E., The, P. S., \& de Winter, D. 1996, A\&A, 309, 809 Barsunova, O. Yu., Grinin, V. P., \& Sergeev, S. G. 2006, Astron. Lett., 32, 924 Bibo, E. A., \& The, P. S. 1990, A\&A, 236, 155

Grady, C. A., Perez, M. R., The, P. S., et al. 1995, A\&A, 302, 472

Grinin, V. P., Kiselev, N. N., Minikulov, N. KH., Chernova, G. P., \& Voshchinnikov, N. V. 1991, A\&SS, 186, 283

Grinin, V. P., The, P. S., de Winter, D., et al. 1994, A\&A, 292, 165

Grinin, V. P., Barsunova, O. Yu., Shugarov, S. Yu., Kroll, P., \& Sergeev, S. G. 2008, Astrophys., 51, 1

Herbst, W., \& Shevchenko, V. S. 1999, AJ, 118, 1043

Herbst, W., Herbst, D. K., Grossman, E. J., \& Weinstein, D. 1994, AJ, 108, 1906 Natta, A., Grinin, V. P., Mannings, V., \& Ungerechts, H. 1997, ApJ, 491, 885

Sandell, G., \& Weintraub, D. A. 2001, ApJS, 134, 115

Semkov, E. H. 2003, A\&A, 404, 655

Semkov, E. H. 2004, A\&A, 419, L59

Semkov, E. H. 2006, AN, 327, 328

Tackett, S., Herbst, W., \& Williams, E. 2003, AJ, 126, 348

Tsvetkov, M. K. 2006, in Virtual Observatory: Plate Content Digitization, Archive Mining and Image Sequence Processing, Heron Press Ltd., Sofia, 10

Yun, J. L., Moreira, M. C., Alves, J. F., \& Storm, J. 1997, A\&A, 320, 167 